\title{
Primes in short arithmetic progressions
}

\author{
by
}

\author{
Jan-Christoph Puchta (Oxford)
}

The large sieve inequality in the form

$$
\sum_{q \leq Q} q \sum_{a=1}^{q}\left|\sum_{\substack{n \leq N \\ n \equiv a(\bmod q)}} a_{n}-\frac{1}{q} \sum_{n \leq N} a_{n}\right|^{2}<\left(N+Q^{2}\right) \sum_{n \leq N}\left|a_{n}\right|^{2}
$$

is essentially optimal. However, in several applications many of the $a_{n}$ vanish, and one might expect better estimates then. In fact, such estimates were given by P. D. T. A. Elliott [1]. He showed the following estimate:

THEOREM 1. Let $N$ and $Q$ be integers, $a_{p}$ be complex numbers for all primes $p \leq N$. Then we have the estimate

$$
\begin{aligned}
& \sum_{q \leq Q}(q-1) \sum_{(a, q)=1}\left|\sum_{\substack{p \leq N \\
p \equiv a(\bmod q)}} a_{p}-\frac{q}{\varphi(q)} \sum_{p \leq N} a_{p}\right|^{2} \\
& \ll_{\varepsilon}\left(\frac{N}{\log N}+Q^{54 / 11+\varepsilon}\right) \sum_{p \leq N}\left|a_{p}\right|^{2} .
\end{aligned}
$$

Under GRH, $Q^{54 / 11}$ may be replaced by $Q^{4+\varepsilon}$. In analogy to the large sieve, he conjectured that one may replace this term by $Q^{2+\varepsilon}$.

Using a completely different approach, Y. Motohashi [4] showed that

$$
\sum_{q \leq Q} \sum_{\chi(\bmod q)}^{*}|\pi(x, \chi)|^{2} \leq \frac{(2+o(1)) x^{2}}{\log x \log \left(x / Q^{1 / 2}\right)}
$$

for $x>Q^{5+\varepsilon}$, where $\pi(x, \chi)=\sum_{p \leq x} \chi(p)$. He also conjectured that $Q^{5+\varepsilon}$ may be replaced by $Q^{2+\varepsilon}$.

Here we will combine the large sieve principle with Selberg's sieve to prove the conjecture of Elliott and give a version of (1) valid for $x>Q^{2+\varepsilon}$.

2000 Mathematics Subject Classification: 11N35, 11N13.

Key words and phrases: large sieve, Selberg's sieve. 
I would like to thank D. R. Heath-Brown for his help with Proposition 9 which allowed me to reduce the exponent to $2+\varepsilon$, and the referee for pointing out some mistakes.

Theorem 2. Let $N$ and $Q$ be integers with $N>Q^{2+\varepsilon}$, $a_{p}$ be complex numbers for any prime $p \leq N$, and let $2 \leq R \leq \sqrt{N}$ be an integer. Then

$$
\sum_{q \leq Q} \sum_{\chi(\bmod q)}^{*}\left|\sum_{p \leq N} a_{p} \chi(p)\right|^{2} \ll \varepsilon \frac{N}{\log N} \sum_{p \leq N}\left|a_{p}\right|^{2} .
$$

As this estimate is the analogue of the large sieve estimate, we can give analogues of Halász-type inequalities, too. As there are a variety of different large value estimates, the same is true for these bounds. However, since the optimal estimate depends on the particular application, we only mention the following:

THEOREM 3. Let $q$ be an integer. Let $\mathcal{C}$ be a set of characters $(\bmod q)$, and $a_{p}$ be complex numbers for any prime $p \leq N$. Then for $k=2,3$ or, if $q$ is cubefree, for any integer $k \geq 2$, we have the estimates

$$
\sum_{\chi \in \mathcal{C}}\left|\sum_{p \leq N} a_{p} \chi(p)\right|^{2} \leq\left(\frac{N}{\log R}+c_{k, \varepsilon} N^{1-1 / k} q^{(k+1) /\left(4 k^{2}\right)+\varepsilon}|\mathcal{C}| R^{2 / k}\right) \sum_{p \leq N}\left|a_{p}\right|^{2}
$$

and

$$
\sum_{\chi \in \mathcal{C}}\left|\sum_{p \leq N} a_{p} \chi(p)\right|^{2} \leq\left(\frac{N}{\log R}+R^{2}|\mathcal{C}| \sqrt{q} \log q\right) \sum_{p \leq N}\left|a_{p}\right|^{2} .
$$

If $\mathcal{C}$ is a set of characters to moduli $q \leq Q$, the same bounds apply with $q$ replaced by $Q^{2}$, where $k$ can be chosen arbitrarily if all occurring values of $q$ are cubefree, and $k=2,3$ otherwise.

From this we conclude immediately

Corollary 4. For $x>Q^{2+\varepsilon}$ we have the estimate

$$
\sum_{q \leq Q} \sum_{\chi(\bmod q)}^{*}|\pi(x, \chi)|^{2} \leq C_{\varepsilon} \frac{x^{2}}{\log ^{2} x} .
$$

Moreover, for $x>Q^{3+\varepsilon}$ this can be made completely explicit:

$$
\sum_{q \leq Q} \sum_{\chi(\bmod q)}^{*}|\pi(x, \chi)|^{2} \leq \frac{(2+o(1)) x^{2}}{\log x \log \left(x / Q^{3}\right)} .
$$

We can also consider a single character:

Corollary 5. Let $\chi$ be a complex character. Then

$$
|\pi(x, \chi)| \leq\left(\left(\frac{1+\phi / \alpha}{2-2 \phi / \alpha}\right)^{1 / 2}+o(1)\right) \frac{x}{\log x},
$$

where $\alpha=\log x / \log q$ and $\phi=1 / 4$ if $q$ is cubefree, and $\phi=1 / 3$ otherwise. 
Note that this estimate is nontrivial if $x>q^{3 / 4}$ resp. $x>q$, depending on whether $q$ is cubefree or not. With a little more work, we obtain the following statement.

Corollary 6. Let $D, x, Q$ be parameters with $x>Q^{1+\varepsilon} D^{2}$. Let $N$ be the number of moduli $q \leq Q$ such that there is some primitive character $\chi$ of order $d \leq D$ and some dth root of unity $\zeta$ such that there is no prime $p \leq x$ with $\chi(p)=\zeta$. Then $N \ll_{\varepsilon} D$.

This was proven by Elliott with $D=3$ under the condition $x>Q^{54 / 11+\varepsilon}$.

We begin the proof of our theorems with the following two sieve principles.

Lemma 7 (Bombieri). Let $V,(\cdot, \cdot)$ be an inner product space, $v_{i} \in V$. Then for any $\Phi \in V$ we have

$$
\sum_{i}\left|\left(\Phi, v_{i}\right)\right|^{2} \leq\|\Phi\|^{2} \max _{i} \sum_{j}\left|\left(v_{i}, v_{j}\right)\right| .
$$

This is Lemma 1.5 of [3].

Lemma 8 (Selberg). Let $R, N$ be integers such that $R^{2}<N$. Then there is a function $g$ which has the following properties:

1. $g(1)=1,|g(n)| \leq 1$ for $n \leq R, g(n)=0$ for $n>R$.

2. $\sum_{n \leq N}((1 * g)(n))^{2} \leq N / \log R+R^{2}$.

This is the usual formulation of Selberg's sieve when used to count the set of primes $\leq N$ (see e.g. [2, Chapter 3, especially Theorem 3.3]). In what follows, we will denote by $g$ the function given by Lemma 8 and set $f=$ $(1 * g)^{2}$. We will have to bound character sums involving $f$; these computations are summarized in the following proposition.

Proposition 9. Let $\chi(\bmod q)$ be a character, $R, N, f$ and $g$ as in Lemma 8, and define $S=\sum_{n \leq N} f(n) \chi(n)$.

1. If $\chi$ is principal, we have $|S|<N / \log R+R^{2}$.

2. Assume that $\chi$ is nonprincipal. Then for any fixed $A$ we have the estimate

$$
\sum_{\nu=1}^{\infty} f(\nu) \chi(\nu) e^{-\log ^{2}(\nu / N)} \ll_{\varepsilon, A} R^{2} q^{1 / 2}\left(\frac{N}{R^{2} q}\right)^{-A} .
$$

3. If $\chi$ is nonprincipal, we have the bounds $|S| \leq R^{2} \sqrt{q} \log q$ and $|S| \leq$ $c_{k, \varepsilon} R^{2 / k} N^{1-1 / k} q^{(k+1) /\left(4 k^{2}\right)+\varepsilon}$ for $k=2,3$, or, if $q$ is cubefree, for $k \geq 2$ arbitrary.

Proof. The first assertion is already contained in Lemma 8. 
Assume now that $\chi$ is nonprincipal. Then

$$
\begin{aligned}
\left|\sum_{n \leq N} f(n) \chi(n)\right| & =\left|\sum_{n \leq N}\left(\sum_{d \mid n} g(d)\right)^{2} \chi(n)\right| \\
& =\left|\sum_{d_{1}, d_{2} \leq R} g\left(d_{1}\right) g\left(d_{2}\right) \chi\left(\left[d_{1}, d_{2}\right]\right) \sum_{n \leq N /\left[d_{1}, d_{2}\right]} \chi(n)\right| \\
& \leq \sum_{d_{1}, d_{2} \leq N}\left|g\left(d_{1}\right) g\left(d_{2}\right)\right| \cdot\left|\sum_{n \leq N /\left[d_{1}, d_{2}\right]} \chi(n)\right| \\
& \leq \sum_{d_{1}, d_{2} \leq R}\left|\sum_{n \leq N /\left[d_{1}, d_{2}\right]} \chi(n)\right| .
\end{aligned}
$$

The inner sum can be estimated using either the Pólya-Vinogradov inequality or Burgess estimates, leading to $|S| \leq R^{2} \sqrt{q} \log q$, resp. $|S| \leq$ $c_{k, \varepsilon} R^{2 / k} N^{1-1 / k} q^{(k+1) /\left(4 k^{2}\right)+\varepsilon}$; thus we obtain the third statement.

To prove the second statement, we begin as above to obtain the inequality

$$
\left|\sum_{n=1}^{\infty} f(n) \chi(n) e^{-\log ^{2}(n / N)}\right| \leq \sum_{d_{1}, d_{2} \leq R}\left|\sum_{n=1}^{\infty} \chi(n) e^{-\log ^{2}\left(\left[d_{1}, d_{2}\right] n / N\right)}\right| .
$$

Write $d=\left[d_{1}, d_{2}\right]$. Using the Mellin transform

$$
\frac{1}{2 \sqrt{\pi} i} \int_{(2)} x^{-s} e^{s^{2} / 4} d s=e^{-\log ^{2} x}
$$

the inner sum can be expressed as

$$
\sum_{n=1}^{\infty} \chi(n) e^{-\log ^{2}(d n / N)}=\frac{1}{2 \sqrt{\pi} i} \int_{(2)} L(s, \chi) e^{s^{2} / 4}(N / d)^{s} d s .
$$

Now we shift the path of integration to the line $\Re s=-A$ with $A>0$. Denote by $\chi_{1}$ the primitive character inducing $\chi$. Then

$$
L(s, \chi)=\prod_{p \mid q_{2}}\left(1-\chi_{1}(p) p^{-s}\right) L\left(s, \chi_{1}\right) .
$$

For $A>2$, the first factor is $\ll q_{2}^{A}$, whereas using the functional equation the $L$-series can be estimated to be $\ll\left(q_{1}(|t|+2)\right)^{A+1 / 2}$, hence the right hand side is

$$
\ll_{A} q^{1 / 2}\left(\frac{N}{d q}\right)^{-A} \leq q^{1 / 2}\left(\frac{N}{R^{2} q}\right)^{-A} .
$$

Hence the whole sum can be bounded by $c(A) R^{2} q^{1 / 2}\left(N /\left(R^{2} q\right)\right)^{-A}$.

To prove Theorem 2, we follow the lines of the proof of the large sieve resp. the Halász inequality; however, we apply Lemma 7 to a different euclidean space. Consider the subspace $V<l^{\infty}$ consisting of all bounded 
sequences $\left(a_{n}\right)$ such that $a_{n}=0$ whenever $f(n)=0$, where $f$ is defined as in Lemma 8. On this space define a product as

$$
\left\langle\left(a_{n}\right),\left(b_{n}\right)\right\rangle:=\sum_{n=1}^{\infty} f(n) e^{-\log ^{2}(n / N)} a_{n} \bar{b}_{n}
$$

Now we apply Lemma 7 to this space and the set of vectors $\Phi=\left(\widehat{a}_{n}\right)$, where $\widehat{a}_{p}=a_{p} e^{\log ^{2}(p / N)}$, for prime numbers $p$ in the range $R^{2}<p \leq N$, and $\widehat{a}_{n}=0$ otherwise, and $v_{i}=(\widehat{\chi}(n))$, where similarly $\widehat{\chi}(n)=\overline{\chi(n)}$, if $f(n) \neq 0$, and 0 otherwise. Now the inequality reads as

$$
\begin{aligned}
& \sum_{q \leq Q} \sum_{\chi(\bmod q)}\left|\sum_{R^{2}<p \leq N} a_{p} \chi(p)\right|^{2} \\
& \leq \max _{\chi}\left(\sum_{n=1}^{\infty} f(n) e^{-\log ^{2}(n / N)}+\sum_{\chi^{\prime} \neq \chi}\left|\sum_{n \leq N} f(n) e^{-\log ^{2}(n / N)} \chi \overline{\chi^{\prime}}(n)\right|\right) \\
& \quad \times \sum_{p \leq N}\left|a_{p}\right|^{2} e^{2 \log ^{2}(p / N)}
\end{aligned}
$$

where the maximum is taken over all characters with moduli at most $Q$. From Lemma 8 it follows that the first term inside the brackets is $\ll N / \log R$ provided that $R<N^{1 / 3}$, say. For the second term, let $\chi$ be a character $(\bmod q)$ and $\chi^{\prime}$ a character $\left(\bmod q^{\prime}\right)$. Then $\chi \overline{\chi^{\prime}}$ is a character $\left(\bmod \left[q, q^{\prime}\right]\right)$. By Proposition 9 , each term in the outer sum can be bounded by $c(A) R^{2}\left[q, q^{\prime}\right]^{1 / 2}$ $\times\left(N /\left(R^{2}\left[q, q^{\prime}\right]\right)\right)^{-A}$, hence the whole sum is $\leq c(A) Q^{3} R^{2}\left(N /\left(R^{2} Q^{2}\right)\right)^{-A}$. Since by assumption $N>Q^{2+\varepsilon}$, we can choose $R=Q^{\varepsilon / 4}, A=6 / \varepsilon+1$ to bound this by some constant depending only on $\varepsilon$. Thus we get the estimate

$$
\sum_{q \leq Q} \sum_{\chi(\bmod q)}\left|\sum_{R^{2}<p \leq N} a_{p} \chi(p)\right|^{2} \ll\left(\frac{N}{\varepsilon \log N}+C_{\varepsilon}\right) \sum_{p \leq N}\left|a_{p}\right|^{2} .
$$

The range $n \leq R^{2}$ can be estimated using the usual large sieve inequality, which gives $\left(R^{2}+Q^{2}\right) \sum_{p \leq N}\left|a_{p}\right|^{2}$, which is negligible. Hence Theorem 2 is proven.

The proof of Theorem 3 is similar, but simpler. First, assume that all characters in $\mathcal{C}$ are characters to a single modulus $q$. We consider the vector space $V<\mathbb{C}^{N}$ consisting of the sequences $\left(a_{n}\right)_{n=1}^{N}$ with $a_{n}=0$ for all $n$ with $f(n)=0$ and the scalar product $\left\langle\left(a_{n}\right),\left(b_{n}\right)\right\rangle:=\sum_{n \leq N} f(n) a_{n} \bar{b}_{n}$. Applying Lemma 7 as above, we obtain the estimate

$$
\sum_{\chi \in \mathcal{C}}\left|\sum_{p \leq N} a_{p} \chi(p)\right|^{2} \leq\left(\frac{N}{\log R}+R^{2}+(|\mathcal{C}|-1) \Delta(R, N, q)\right) \sum_{R \leq p \leq N}\left|a_{p}\right|^{2}
$$


where $\Delta(R, N, q)$ is the bound obtained by Proposition 9 , i.e. $\Delta(R, N, q) \leq$ $R^{2} \sqrt{q} \log q$, resp. $\Delta(R, N, q)<c_{k, \varepsilon} q^{(k+1) /\left(4 k^{2}\right)+\varepsilon} N^{1-1 / k} R^{2 / k}$. The term $R^{2}$ can be neglected in comparison with $\Delta(R, N, q)$. This is obvious in the first case. In the second case, we may assume that $\Delta(R, N, q)<N$, since otherwise Theorem 3 is an immediate consequence of the Cauchy-Schwarz inequality. This implies $R<N^{1 / 2} q^{-(k+1) /(2 k)}$, which in turn implies

$$
R^{2}<N^{1-1 / k} q^{-1-1 / k}<\Delta(R, N, q) .
$$

Hence we obtain Theorem 3 for sets of characters belonging to a single modulus.

The proof for the case that the characters belong to different moduli is similar; note that $\left[q_{1}, q_{2}\right]$ is cubefree if both $q_{1}$ and $q_{2}$ are cubefree.

In the range $Q^{2+\varepsilon} \leq x<Q^{3+\varepsilon}$, Corollary 4 follows from Theorem 2 by choosing $a_{p}=1$ for all prime numbers $p \leq N$, whereas in the range $x>Q^{3+\varepsilon}$ it follows from Theorem 3 . Similarly we obtain Corollary 5 from Theorem 3. We choose $\mathcal{C}=\left\{\chi_{0}, \chi, \bar{\chi}\right\}$ to obtain the estimate

$$
|\pi(x)|^{2}+2|\pi(x, \chi)|^{2} \leq \frac{x}{\log \left(c_{k, \varepsilon} x^{1 / 2} q^{(k+1) /(8 k)+\varepsilon}\right)} \pi(x)
$$

and choosing either $k=3$ or $k \nearrow \infty$ we obtain the result by solving for $|\pi(x, \chi)|$.

To prove Corollary 6 , let $\mathcal{P}$ be the set of prime numbers $p$, such that there is some character $\chi$ of order $d$ as described in the corollary. For every such $p$, choose such a character $\chi_{1}$ together with all its powers, and denote the set of all these characters with $\mathcal{C}$. Let $\zeta$ be a $d$ th root of unity. We have

$$
\sum_{\substack{\chi^{d}=\chi_{0} \\ \chi \neq \chi_{0}}}|\pi(x, \chi)|^{2}=d \sum_{a=1}^{d}\left|\#\left\{p \leq x \mid \chi_{1}(p)=\zeta^{a}\right\}-\frac{1}{d} \pi\left(x, \chi_{0}\right)\right|^{2} .
$$

Since by assumption, one of the terms on the right hand side is large, the right hand side is

$$
\gg \frac{x^{2}}{d \log ^{2} x} \geq \frac{x}{D \log ^{2} x} .
$$

Now we have $|\mathcal{C}| \leq D \cdot|\mathcal{P}|$; thus we get

$$
|\mathcal{P}| \frac{x^{2}}{D \log ^{2} x} \ll \frac{x^{2}}{\log x \log R}+x D R^{2}|\mathcal{P}| Q \log Q .
$$

If $D^{2} Q \log Q<x^{1-\varepsilon}$, we can choose $R=x^{\varepsilon / 4}$, and the second term on the right hand side is still of lesser order than the left hand side. With this choice the inequality can be simplified to $|\mathcal{P}| \ll_{\varepsilon} D$. 


\section{References}

[1] P. D. T. A. Elliott, Subsequences of primes in residue classes to prime moduli, in: Studies in Pure Mathematics to the Memory of P. Turán, P. Erdős (ed.), Akadémiai Kiadó, Budapest, 1983, 157-164.

[2] H. Halberstam and H.-E. Richert, Sieve Methods, London Math. Soc. Monographs 4, Academic Press, 1974.

[3] H. L. Montgomery, Topics in Multiplicative Number Theory, Lecture Notes in Math. 227, Springer, 1971.

[4] Y. Motohashi, Large sieve extensions of the Brun-Titchmarsh theorem, in: Studies in Pure Mathematics to the Memory of P. Turán, P. Erdős (ed.), Akadémiai Kiadó, Budapest, 1983, 507-515.

Mathematical Institute

University of Oxford

24-29 St. Giles' Street

Oxford, OX1 3LB, U.K.

E-mail: puchta@maths.ox.ac.uk
Current address:

Mathematisches Institut

Eckerstr. 1

79111 Freiburg, Germany

E-mail: jcp@arcade.mathematik.uni-freiburg.de

Received on 13.6.2001

and in revised form on 22.4.2002 